\title{
Author index \\ Volume 25
}

Aceves, A. B., see Castro-Castro, C.

Ahmad Hambali, N. A. M., see Zakiah Malek, A.

25 (2016) 1650042

25 (2016) 1650049

25 (2016) 1650025

Ahmad, H., see Ismail, E. I.

Ahmad, H., see Latiff, A. A.

25 (2016) 1650034

Ajiya, M., Oldapo, J. A. \& Hambali, N. A. M. A., Lasing

threshold characteristics of multi-wavelength Brillouin-erbium

laser in the L-band region assisted by delay interferometer

25 (2016) 1650024

Akhlaghi, M., see Kaboli, $M$.

Alberucci, A., see Smyth, N. F.

Alexandar, A., Surendran, P., Sakthy Priya, S., Lakshmanan, A.

\& Rameshkumar, P., Growth and characterizations of

L-methioninium picrate single crystal for nonlinear optical applications

25 (2016) 1650018

25 (2016) 1650043

Alexandar, A., Surendran, P., Sakthy Priya, S., Lakshmanan, A.

\& Rameshkumar, P., Studies on growth and characterization of nonlinear optical L-tartaric acid-nicotinamide single crystal

Allam, S. R., see Chakravarthy, G.

Alvarez-Ortiz, M., see Salazar-Romero, M. Y.

An, N., Ren, H. J., Deng, X. W., Zhao, X. H., Gao, Y. Q., Xu, Z. Y., Rao, D. X., Cui, Y., Ji, L. L., Zhao, Z. X., Liu, D., Wang, T., Chen, M., Xia, L., Feng, W., Cao, Z. D., Yang, X. D., Ma, W. X. \& Chen, X. F., The role of ferroelectric domain wall in nonlinear cerenkov frequency up-conversion in $1 D$ nonlinear crystal

Arzola, A. V., see Salazar-Romero, M. Y.

Asadpour, S. H., see Solookinejad, Gh.

Asadpour, S. H., see Solookinejad, G.

Assanto, G. \& Smyth, N. F., Nonlinear guided waves: Preface

Assanto, G., see Smyth, N. F.

Ayala, Y. A., see Salazar-Romero, M. Y.

Azmi, A. \& Marchant, T. R., Circular dispersive shock waves in colloidal media

Azwar, A., Soehianie, A., Iskandar, A. A. \& Tjia, M.-O.,

Fano-like spectral profile in TE wave scattering by nanowire of dissipative and dispersive materials

Bai, R. P., see Gu, X. T.

Bai, R., Jin, X. R., Zhang, Y. Q., Zhang, S. \& Lee, Y., Narrow-dual-band perfect absorption plasmonic sensor in metamaterials based on the coupling of two resonators 
Balbuena Ortega, A., see Salazar-Romero, M. Y.

25 (2016) 1650048

Bhattacharya, S., see Panda, $R$.

25 (2016) 1650029

Boudebs, G., see Chniti, $M$.

25 (2016) 1650020

Boudebs, G., see Chniti, M.

25 (2016) 1650050

Cao, H., see Wang, $X$.

Cao, Z. D., see $A n, N$.

Cárdenas, J. F., see Paz, J. L.

Cassagne, C., see Chniti, $M$.

Cassagne, C., see Chniti, $M$.

Castle, R.-S., see Liu, H.

Castro, C. J. \& Urzagasti, D., Seesaw drift of bright solitons of the nonlinear Schrödinger equation with a periodic potential

Castro-Castro, C., Shen, Y., Srinivasan, G., Aceves, A. B. \& Kevrekidis, P. G., Light dynamics in nonlinear trimers and twisted multicore fibers

25 (2016) 1650014

25 (2016) 1650008

25 (2016) 1650016

25 (2016) 1650020

25 (2016) 1650050

25 (2016) 1650053

25 (2016) 1650038

Chakravarthy, G., Allam, S. R., Sharan, A., Ghosh, O. S. N., Gayathri, S., Viswanath, A. K., Prabhakar, M. N. \& Song, J.-I., Understanding the effect of bound excitons on two photon absorption process in anatase $\mathrm{TiO}_{2}$ nanospheres using ultrafast pulses

25 (2016) 1650019

Chen, G., Wang, H., Fan, L. \& Liu, Y., Discrete solitons in an array of linear waveguides with a nonlinear $\mathscr{P} \mathscr{T}$-symmetric defect

25 (2016) 1650026

25 (2016) 1650008

Chen, M., see $A n, N$.

Chen, X. F., see $A n, N$.

Chniti, M., Cassagne, C., Wang, H. \& Boudebs, G., Dark-field 25 (2016) 1650008 Z-scan technique with highly nonlinear absorbing materials: Application to porphyrins

25 (2016) 1650020

Chniti, M., Cassagne, C., Wang, H. \& Boudebs, G., Nonlinear properties of unfilled d shell metal porphyrins of 5, 10, 15, 20-tetraphenyl-21H, 23H-porphine cobalt(II) et 5, 10, 15, 20-tetrakis(4-methoxyphenyl)-21H, 23H-porphine cobalt(II) using D4 $\sigma$-Z-scan

Cicili Ignatius, I., Rajathi, S., Kirubavathi, K. \& Selvaraju, K., Enhancement of second harmonic generation efficiency, laser damage threshold and optical properties of cobalt chloride doped with L-alanine single crystal

Costa-Vera, C., see Paz, J. L.

Cui, Y., see $A n, N$.

Dar, M. H., see Srinivasa Rao, A.

Das, S. K., see Panda, $R$.

Datta, P. K., see Panda, R.

Deng, X. W., see An, N.

Dey, S. \& Mukhopadhyay, S., All-optical high frequency clock pulse generator using the feedback mechanism in Toffoli gate with Kerr material

Du, J. Q., see Li, L.

Entezar, S. R. \& Vatannejad, R., 1D graded thickness nonlinear structure as an optical diode

25 (2016) 1650050

Fan, L., see Chen, G.

25 (2016) 1650017

25 (2016) 1650016

25 (2016) 1650008

25 (2016) 1650039

25 (2016) 1650029

25 (2016) 1650029

25 (2016) 1650008

25 (2016) 1650012

25 (2016) 1650009

25 (2016) 1650030

25 (2016) 1650026 
Feng, L., see Liu, $H$.

25 (2016) 1650053

Feng, W., see $A n, N$.

25 (2016) 1650008

Gao, H., see Wang, X.

25 (2016) 1650014

Gao, Y. Q., see An, N.

25 (2016) 1650008

Gayathri, S., see Chakravarthy, G.

Ghosh, O. S. N., see Chakravarthy, G.

25 (2016) 1650019

25 (2016) 1650019

Gong, Z., see $L v, Z$.

Goyal, A., see Pal, $R$.

Gu, J., see Wang, $X$.

Gu, X. T., Bai, R. P., Jin, X. R., Zhang, Y. Q. \& Zhang, S., Ultra-narrow-band perfect absorber based on high-order plasmonic resonance in metamaterial

Guo, X.-Z., Liu, C., Zhou, Y. \& Luo, D.-B., All-optical logical gates based on photoinduced molecules reorientation in amorphous polymer films

Hambali, N. A. M. A., see Ajiya, M.

Harun, S. W., see Ismail, E. I.

Harun, S. W., see Latiff, A. A.

Hassan, S. S., see Sharaby, Y. A.

Hatami, M., see Pakarzadeh, $H$.

He, W., see Wang, $X$.

Hernández-Cordero, J., see Salazar-Romero, M. Y.

Herrera, R. A. \& Rodriguez, C. A., Dependence of the shape of fiber tapers on the nonlinear pulse propagation

Hu, D., Wang, H.-Y., Zhu, Q.-F., Zhang, X.-W. \& Tang, Z.-J., Investigation of a broadband and polarization-insensitive optical absorber based on closed-ring resonator

$\mathrm{Hu}, \mathrm{H}$., see Wang, $X$.

Iskandar, A. A., see Azwar, A.

Iskandar, A. A., see Widiasari, F. R.

Ismail, E. I., Kadir, A., Latiff, A. A., Ahmad, H. \& Harun, S. W., Q-switched erbium-doped fiber laser operating at $1502 \mathrm{~nm}$ with molybdenum disulfide saturable absorber

Iza, P., see Rodriguez, L. G.

Ji, L. L., see $A n, N$.

Jin, X. R., see Bai, R.

Jin, X. R., see Gu, X. T.

Kaboli, M. \& Akhlaghi, M., Optical nonlinear enhancement from binary optimization of plasmonic nano-bi-domes

Kadir, A., see Ismail, E. I.

Karpov, D., Scherbak, S., Svirko, Y. \& Lipovskii, A., Second harmonic generation from hemispherical metal nanoparticle covered by dielectric layer

Kaur, S., All-optical binary full subtractor using logic operations based on nonlinear properties of semiconductor optical amplifier

25 (2016) 1650028

25 (2016) 1650033

25 (2016) 1650014

25 (2016) 1650011

25 (2016) 1650004

25 (2016) 1650024

25 (2016) 1650025

25 (2016) 1650034

25 (2016) 1650021

25 (2016) 1650023

25 (2016) 1650014

25 (2016) 1650048

25 (2016) 1650002

25 (2016) 1650032

25 (2016) 1650014

25 (2016) 1650005

25 (2016) 1650006

25 (2016) 1650025

25 (2016) 1650022

25 (2016) 1650008

25 (2016) 1650027

25 (2016) 1650011

25 (2016) 1650018

25 (2016) 1650025

25 (2016) 1650001

25 (2016) 1650003

25 (2016) 1650007

25 (2016) 1650042

25 (2016) 1650054

25 (2016) 1650017

Kirubavathi, K., see Cicili Ignatius, I. 
Kumar, C. N., see Pal, R.

Lakshmanan, A., see Alexandar, A.

Lakshmanan, A., see Alexandar, A.

Latiff, A. A., Shamsudin, H., Tiu, Z. C., Ahmad, H. \& Harun, S. W., Switchable soliton mode-locked and multi-wavelength operation in thulium-doped all-fiber ring laser

Latiff, A. A., see Ismail, E. I.

Lee, Y., see Bai, $R$.

Lei, D. J., see Zhang, J. G.

Li, L., Zhang, B., Du, J. Q., Zhang, Z. G., Wang, D. X. \& Zhang, X. L., Bistable upconversion emission in Yb-sensitized Tm: $\mathrm{ZrO}_{2}$ nanophosphors at room temperature

Li, W., see Liu, $H$.

Li, Y. F., see Zhang, J. G.

Lipovskii, A., see Karpov, D.

Liu, C., see Guo, X.-Z.

Liu, D., see $A n, N$.

Liu, H., Castle, R.-S., Li, W. \& Feng, L., Spatial distribution and quantum trajectory control of the molecular harmonic spectra

Liu, Y., see Chen, G.

Loomba, S., see Pal, R.

$\mathrm{Lu}, \mathrm{X}$., see $L v, Z$.

Luo, D.-B., see Guo, X.-Z.

Lv, Z., Wu, J., Gong, Z., Lu, X. \& Shi, K., Spectrally tunable femtosecond erbium fiber laser based on large-normal-dispersion cavity

Lynch, S., see Sharaby, Y.A.

Ma, W. X., see An, N.

Mandal, S., see Verma, $N$.

Marchant, T. R., see Azmi, A.

Marlow, F., see Muldarisnur, $M$.

Martínez-Galicia, R. \& Panayotaros, P., Coherent states and localization in a quantized discrete NLS lattice

Miladinović, T. B., see Petrović, V. M.

Morita, K., see Suherman

Mukhopadhyay, S., see Dey, S.

Muldarisnur, M. \& Marlow, F., Polarization angular-resolved transmission spectroscopy of opal films

Nath, D. \& Roy, P., Exact localized solutions of $(1+1)$-dimensional nonlinear Schrödinger equation with complex $\mathcal{P} \mathcal{T}$ symmetric potentials and power-law nonlinearity

Oldapo, J. A., see Ajiya, $M$.

Pakarzadeh, H., Taghizadeh, M. \& Hatami, M., Designing a photonic crystal fiber for an ultra-broadband parametric amplification in telecommunication region

25 (2016) 1650033

25 (2016) 1650037

25 (2016) 1650052

25 (2016) 1650034

25 (2016) 1650025

25 (2016) 1650027

25 (2016) 1650010

25 (2016) 1650009

25 (2016) 1650053

25 (2016) 1650010

25 (2016) 1650001

25 (2016) 1650004

25 (2016) 1650008

25 (2016) 1650053

25 (2016) 1650026

25 (2016) 1650033

25 (2016) 1650028

25 (2016) 1650004

25 (2016) 1650028

25 (2016) 1650021

25 (2016) 1650008

25 (2016) 1650013

25 (2016) 1650044

25 (2016) 1650015

25 (2016) 1650047

25 (2016) 1650040

25 (2016) 1650007

25 (2016) 1650012

25 (2016) 1650015

25 (2016) 1650036

25 (2016) 1650024

25 (2016) 1650023

Pal, R., Goyal, A., Loomba, S., Raju, T. S. \& Kumar, C. N., Compression of optical similaritons induced by cubic-quintic nonlinear media in a graded-index waveguide

25 (2016) 1650033

Panahi, M., see Solookinejad, Gh.

25 (2016) 1650035

Panahi, M., see Solookinejad, G.

25 (2016) 1650031 
Panayotaros, P., see Martínez-Galicia, $R$.

25 (2016) 1650047

Panayotaros, P., Shelf solutions and dispersive shocks in a discrete NLS equation: Effects of nonlocality

Panda, R., Bhattacharya, S., Samal, R., Singh, A., Sahoo, P. K.,

25 (2016) 1650045

Datta, P. K. \& Das, S. K., Second harmonic generation of femtosecond pulses using $\mathrm{ZnO}$ nanorods grown by chemical bath deposition with drop casted seed layer

25 (2016) 1650029

Paz, J. L., Rodríguez, L. G., Cárdenas, J. F. \& Costa-Vera, C., Solvent effects in the nonlinear optical properties using the Voigt function

Paz, J. L., see Rodriguez, L. G.

Petrović, V. M. \& Miladinović, T. B., Kinetic energy distribution of photoelectrons in the tunnel ionization process in the case of ultrashort laser pulses

Piccardi, A., see Smyth, N. F.

Prabhakar, M. N., see Chakravarthy, G.

Quy, H. Q., see Trung, T. D.

Rajathi, S., see Cicili Ignatius, I.

Raju, T. S., see Pal, R.

Rameshkumar, P., see Alexandar, A.

Rameshkumar, P., see Alexandar, A.

Rao, D. X., see $A n, N$.

Ren, H. J., see $A n, N$.

Rodríguez, L. G., see Paz, J. L.

Rodriguez, C. A., see Herrera, R. A.

Rodriguez, L. G., Iza, P. \& Paz, J. L., Study of dependence between thermal diffusivity and sample concentration measured by means of frequency-resolved thermal lens experiment

Roy, P., see Nath, D.

Sahoo, P. K., see Panda, $R$.

Sakthy Priya, S., see Alexandar, A.

Sakthy Priya, S., see Alexandar, A.

Salazar-Romero, M. Y., Alvarez-Ortiz, M., Balbuena Ortega, A., Ayala, Y. A., Arzola, A. V., Hernández-Cordero, J. \& Volke-Sepulveda, K., Nonlinear optical properties of dielectric nanocolloids: Particle size and concentration effects

Samal, R., see Panda, $R$.

Sangachin, E. A., see Solookinejad, Gh.

Sangachin, E. A., see Solookinejad, G.

Scherbak, S., see Karpov, D.

Selvaraju, K., see Cicili Ignatius, I.

Shahimin, M. M., see Zakiah Malek, A.

Shamsudin, H., see Latiff, A. A.

Sharaby, Y. A., Lynch, S. \& Hassan, S. S., Inhomogeneous and transverse field effects on time delayed optical bistability inside and outside the rotating wave approximation

Sharan, A., see Chakravarthy, G.

Sharan, A., see Srinivasa Rao, A.

Shen, Y., see Castro-Castro, $C$.

Shi, K., see $L v, Z$.

25 (2016) 1650016

25 (2016) 1650022

25 (2016) 1650040

25 (2016) 1650043

25 (2016) 1650019

25 (2016) 1650054

25 (2016) 1650017

25 (2016) 1650033

25 (2016) 1650037

25 (2016) 1650052

25 (2016) 1650008

25 (2016) 1650008

25 (2016) 1650016

25 (2016) 1650002

25 (2016) 1650022

25 (2016) 1650036

25 (2016) 1650029

25 (2016) 1650037

25 (2016) 1650052

25 (2016) 1650048

25 (2016) 1650029

25 (2016) 1650035

25 (2016) 1650031

25 (2016) 1650001

25 (2016) 1650017

25 (2016) 1650049

25 (2016) 1650034

25 (2016) 1650021

25 (2016) 1650019

25 (2016) 1650039

25 (2016) 1650042

25 (2016) 1650028 
Singh, A., see Panda, $R$.

25 (2016) 1650029

Smyth, N. F. \& Tope, B., Beam on beam control: Beyond the particle approximation

25 (2016) 1650046

Smyth, N. F., Piccardi, A., Alberucci, A. \& Assanto, G., Highly nonlocal optical response: Benefit or drawback?

Smyth, N. F., see Assanto, G.

Soehianie, A., see Azwar, A.

Solookinejad, G., Panahi, M., Sangachin, E. A. \& Asadpour, S. H., Plasmonic nanostructure induced simultaneous slow and fast light propagation in a slab doped by four-level quantum dots

Solookinejad, Gh., Panahi, M., Sangachin, E. A. \& Asadpour, S. H., Spin coherence control of optical bistability via light hole transition in multiple quantum well waveguide embedded in a dielectric medium

Song, J.-I., see Chakravarthy, G.

Srinivasa Rao, A., Dar, M. H., Venkatramaiah, N., Venkatesan, R. \& Sharan, A., Third order optical nonlinear studies and its use to estimate thickness of sandwiched films of tetra-phenyl porphyrin derivatives

Srinivasan, G., see Castro-Castro, C.

Suherman Morita, K. \& Kawaguchi, T., Surface plasmon resonance sensor using functionalized alkanethiols monolayer for illegal compound detection

Surendran, P., see Alexandar, A.

Surendran, P., see Alexandar, A.

Svirko, Y., see Karpov, D.

Taghizadeh, M., see Pakarzadeh, $H$.

Tang, Z.-J., see $H u, D$.

Tiu, Z. C., see Latiff, A. A.

Tjia, M.-O., see Azwar, A.

Tjia, M.-O., see Widiasari, F. R.

Tope, B., see Smyth, N. F.

Trung, T. D., Kien, B. X., Tung, N. T. \& Quy, H. Q., Dynamics of polystyrene beads linking to DNA molecules under single optical tweezers: A numerical study using full normalized Langevin equation

Tung, N. T., see Trung, T. D.

Urzagasti, D., see Castro, C. J.

Valovik, D., Novel propagation regimes for TE waves guided by a waveguide filled with Kerr medium

Vatannejad, R., see Entezar, S. R.

Venkatesan, R., see Srinivasa Rao, A.

Venkatramaiah, N., see Srinivasa Rao, A.

Verma, N. \& Mandal, S., Performance analysis of optical micro-ring resonator as all-optical reconfigurable logic and multiplexer in Z-domain

Viswanath, A. K., see Chakravarthy, G.

Volke-Sepulveda, K., see Salazar-Romero, M. Y.

Wahid, M. H. A., see Zakiah Malek, A.

Wang, D. X., see Li, L.

25 (2016) 1650043

25 (2016) 1650041

25 (2016) 1650005

25 (2016) 1650031

25 (2016) 1650035

25 (2016) 1650019

25 (2016) 1650039

25 (2016) 1650042

25 (2016) 1650007

25 (2016) 1650037

25 (2016) 1650052

25 (2016) 1650001

25 (2016) 1650023

25 (2016) 1650032

25 (2016) 1650034

25 (2016) 1650005

25 (2016) 1650006

25 (2016) 1650046

25 (2016) 1650054

25 (2016) 1650054

25 (2016) 1650038

25 (2016) 1650051

25 (2016) 1650030

25 (2016) 1650039

25 (2016) 1650039

25 (2016) 1650013

25 (2016) 1650019

25 (2016) 1650048

25 (2016) 1650049

25 (2016) 1650009 
Wang, D., see Wang, $X$.

Wang, H., see Chen, G.

Wang, H., see Chniti, $M$.

Wang, H., see Chniti, $M$.

Wang, H., see Wang, $X$.

Wang, H.-Y., see Hu, D.

Wang, T., see An, N.

Wang, X., Wang, D., Gao, H., Yang, Z., Cao, H., Yang, H., He, W., Wang, H., Gu, J. \& Hu, H., Third-order nonlinear optical properties of a novel series of $D-\pi-A$ pyrene-aldehyde

derivatives

Widiasari, F. R., Iskandar, A. A. \& Tjia, M.-O., Validity of classical coupled oscillators model for elucidating surface plasmon effects on optical scattering by silver nanocylinders

$\mathrm{Wu}$, J., see $L v, Z$.

Xia, L., see $A n, N$.

Xiang, Y. J., see Zhang, J. G.

$\mathrm{Xu}, \mathrm{Z}$. Y., see $A n, N$.

Yang, H., see Wang, $X$.

Yang, X. D., see An, $N$.

Yang, Z., see Wang, $X$.

Zakiah Malek, A., Ahmad Hambali, N. A. M., Wahid, M. H. A. \& Shahimin, M. M., Lasing characteristics of $10 \mathrm{GHz}$ signal spacing in a ring cavity multi-wavelength Brillouin fiber laser employing fiber Bragg grating

Zhang, B., see $L i, L$.

Zhang, J. G., Xiang, Y. J., Li, Y. F., Lei, D. J. \& Zhang, L. F., Role of anomalous self-steepening in controlling self-accelerating Airy pulses in nonlinear metamaterials Zhang, L. F., see Zhang, J. G.

Zhang, S., see Bai, $R$.

Zhang, S., see Gu, X. T.

Zhang, X. L., see Li, $L$.

Zhang, X.-W., see Hu, D.

Zhang, Y. Q., see Bai, $R$.

Zhang, Y. Q., see Gu, X. T.

Zhang, Z. G., see Li, L.

Zhao, X. H., see $A n, N$.

Zhao, Z. X., see An, N.

Zhou, Y., see Guo, X.-Z.

Zhu, Q.-F., see Hu, D.
25 (2016) 1650014

25 (2016) 1650026

25 (2016) 1650020

25 (2016) 1650050

25 (2016) 1650014

25 (2016) 1650032

25 (2016) 1650008

25 (2016) 1650014

25 (2016) 1650006

25 (2016) 1650028

25 (2016) 1650008

25 (2016) 1650010

25 (2016) 1650008

25 (2016) 1650014

25 (2016) 1650008

25 (2016) 1650014

25 (2016) 1650049

25 (2016) 1650009

25 (2016) 1650010

25 (2016) 1650010

25 (2016) 1650027

25 (2016) 1650011

25 (2016) 1650009

25 (2016) 1650032

25 (2016) 1650027

25 (2016) 1650011

25 (2016) 1650009

25 (2016) 1650008

25 (2016) 1650008

25 (2016) 1650004

25 (2016) 1650032 